\title{
Some theorems and properties of multi-dimensional fractional Laplace transforms
}

\begin{abstract}
The aim of this work is to study theorems and properties for the one-dimensional fractional Laplace transform, generalize some properties for the one-dimensional fractional Lapalce transform to be valid for the multi-dimensional fractional Lapalce transform and is to give the definition of the multi-dimensional fractional Lapalce transform. This study includes: dedicate the one-dimensional fractional Laplace transform for functions of only one independent variable with some of important theorems and properties and develop of some properties for the one-dimensional fractional Laplace transform to multi-dimensional fractional Laplace transform. Also, we obtain a fractional Laplace inversion theorem after a short survey on fractional analysis based on the modified Riemann-Liouville derivative.
\end{abstract}

Keyword: Theorems; Properties; Multi-dimensional fractional Laplace transforms 